EPJ Web of Conferences 59, 05006 (2013)

DOI: $10.1051 /$ epjconf/20135905006

(C) Owned by the authors, published by EDP Sciences, 2013

\title{
Laser-plasma interaction physics for shock ignition
}

\author{
C. Goyon ${ }^{1}$, S. Depierreux ${ }^{1}$, D.T. Michel ${ }^{1}$, G. Loisel ${ }^{2}$, V. Yahia ${ }^{2}$, \\ P.E. Masson-Laborde ${ }^{1}$, P. Loiseau ${ }^{1}$, S. Hüller ${ }^{3}$, N.G. Borisenko ${ }^{4}$, A. Orekhov ${ }^{4}$, \\ O. Rosmej ${ }^{6}$, P. Nicolaï ${ }^{5}$, V.T. Tikhonchuk ${ }^{5}$ and C. Labaune ${ }^{2}$
}

${ }^{1}$ CEA, DAM, DIF, 91297 Arpajon, France

${ }^{2}$ LULI, Ecole Polytechnique, CNRS, CEA, UPMC, 91128 Palaiseau, France

${ }^{3}$ Centre de Physique Théorique, Ecole Polytechnique, 91128 Palaiseau Cedex, France

${ }^{4}$ Lebedev Physical Institute, 53 Leninskii Prospect, 119991 Moscow, Russia

${ }^{5}$ CELIA, Université Bordeaux 1, 351 cours de la Libération, 33405 Talence Cedex, France

${ }^{6}$ GSI Helmhotzzentrum fur Schwerionenforschung, 64291 Darmstadt, Germany

\begin{abstract}
In the shock ignition scheme, the ICF target is first compressed with a long (nanosecond) pulse before creating a convergent shock with a short $(\sim 100 \mathrm{ps})$ pulse to ignite thermonuclear reactions. This short pulse is typically $\left(\sim 2.10^{15}-10^{16} \mathrm{~W} / \mathrm{cm}^{2}\right)$ above LPI (Laser Plasma Instabilities) thresholds. The plasma is in a regime where the electron temperature is expected to be very high $(2-4 \mathrm{keV})$ and the laser coupling to the plasma is not well understood. Emulating LPI in the corona requires large and hot plasmas produced by high-energy lasers. We conducted experiments on the LIL (Ligne d'Integration Laser, $10 \mathrm{~kJ}$ at $3 \omega)$ and the LULI2000 $(0.4 \mathrm{~kJ}$ at $2 \omega)$ facilities, to approach these conditions and study absorption and LPI produced by a high intensity beam in preformed plasmas. After introducing the main risks associated with the short pulse propagation, we present the latest experiment we conducted on LPI in relevant conditions for shock ignition.
\end{abstract}

\section{EXPERIMENTAL CONTEXT}

Shock ignition (SI) $[1,2]$ is considered as a relatively robust way to achieve efficient fuel burn. SI is a two-step concept with a spherical compression separated from the ignition phase which uses a strong convergent shock. This shock is launched at the end of the compression stage using a $10^{15}$ $10^{16} \mathrm{~W} / \mathrm{cm}^{2}$ laser spike. When this shock collides with the return shock near the inner shell surface, it creates a third converging shock that leads to ignition. One of the principal questions regarding this scheme concerns the propagation and absorption of the spike in the plasma corona surrounding the fuel. As shown in [6], for an electron temperature (at the end of compression) $\mathrm{Te}=4 \mathrm{keV}$, a gradient density scale length of $750 \mu \mathrm{m}$ and a $3 \omega$ pulse for the spike, LPI intensity thresholds are $2 \times 10^{15} \mathrm{~W} / \mathrm{cm}^{2}$ for Stimulated Brillouin Scattering (SBS), $1.7 \times 10^{15} \mathrm{~W} / \mathrm{cm}^{2}$ for Stimulated Raman Scattering (SRS) and $8 \times 10^{13} \mathrm{~W} / \mathrm{cm}^{2}$ for Two Plasmon Decay (TPD). That means the SI spike intensity lies above these thresholds where LPI can lead to significant effects. Concerning the absorption mechanism, Inverse Bremsstrahlung absorption is dominant at the compression stage $[7,8]$ but studies showed that collisionless absorption mechanisms may be induced by the spike [9].

Previous experiments studied the propagation and absorption over a wide range of laser intensities through a long and hot plasma [10]. For conditions relevant to the SI scheme, it showed strong filamentation of the beam that would reduce the shock efficiency. It also evidenced beneficial effect of beam smoothing techniques and the use of longer plasma (with greater density scalelength $\mathrm{L}_{\mathrm{ne}}$ ) leading

This is an Open Access article distributed under the terms of the Creative Commons Attribution License 2.0, which permits unrestricted use, distribution, and reproduction in any medium, provided the original work is properly cited. 


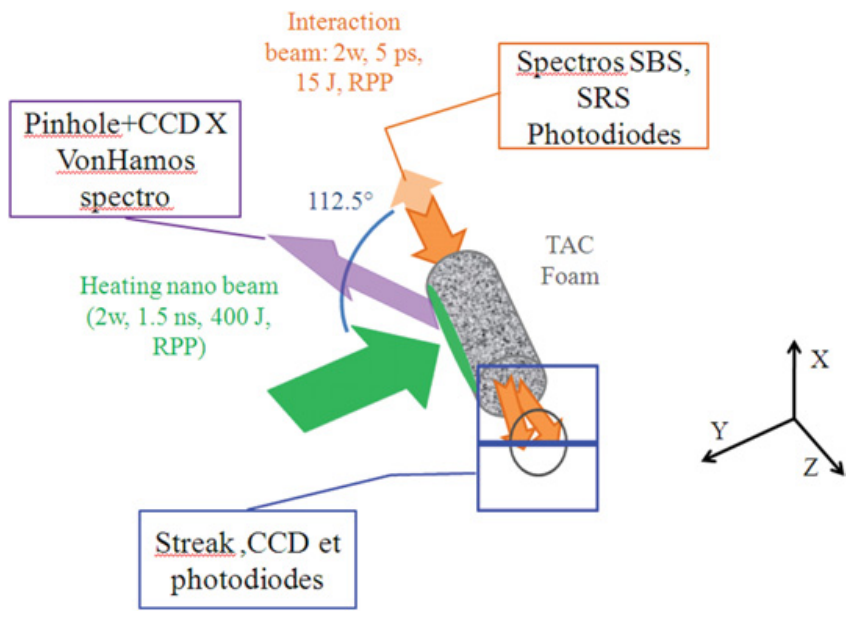

Figure 1. Experimental Scheme.

to better collisional absorption. Concerning the backscattering instabilities, it emphasized that despite huge linear gains $(\geq 20)$ SBS reflectivity was less than $10 \%$ and that SRS reflectivity was already at the percent level for very small linear gain $(\approx 2)$. These previous results were obtained with a single speckle. On the LULI2000, we used a Random Phase Plate (RPP) to control intensity (relevant for SI scheme) and speckle distribution and studied if the filamentation was still spreading the overall distribution. After a presentation of the experimental setup, we will present the plasma conditions and the experimental results on filamentation.

\section{EXPERIMENTAL CAMPAIGN ON THE LULI2000}

\subsection{Experimental scheme}

As the results obtained on the LIL facility have been detailed in $[2-4,11]$, we will focus on the last experiment we conducted on the LULI2000. We used a $1.5 \mathrm{~ns} 2 \omega 0.4 \mathrm{~kJ}$ laser beam (creation beam) to ionize the targets and to obtain the exponential density profile which is typical for the corona at the end of the compression stage. Then, one nanosecond later, we send the $5 \mathrm{ps}, 2 \omega 15 \mathrm{~J}$ beam (interaction beam) focused with an f/4 off axis parabola. Figure 1 presents a sketch of the experiment and the main diagnostics. The targets were TAC (cellulose triacetate) foams of different densities with length (along the picosecond beam direction $\mathrm{z}$ ) varying from $300 \mu \mathrm{m}$ to $1 \mathrm{~mm}$. To heat homogeneously the total length of the foam (despite the $112.5^{\circ}$ angle between the picosecond and the nanosecond beam), we used a Random Phase Plate (RPP) with rectangular elements on the nanosecond beam to have a focal spot on target of approximately $100 \mu \mathrm{m} \times \mathrm{L}(\mu \mathrm{m})$ where $\mathrm{L}$ is the length of the foam. At best focus ( $15 \mathrm{~J}$ at $2 \omega$ on $20 \mu \mathrm{m}$ diameter focal spot), we can reach $\mathrm{I}_{\max }=10^{18} \mathrm{~W} / \mathrm{cm}^{2}$ on the picosecond beam, that's why we can use a RPP to control the intensity distribution on target and still achieve SI relevant intensity. On each shot, we used two spectrometers, one with high spectral resolution for SBS, and one with high spectral range (from $500 \mathrm{~nm}$ to $1100 \mathrm{~nm}$ ) for SRS, both were coupled with streak cameras $\left(\Delta \lambda_{\mathrm{SBS}}=5.8 \times 10^{-3} \mathrm{~nm} / \mathrm{pix}, \Delta \lambda_{\mathrm{SRS}}=0.61 \mathrm{~nm} / \mathrm{pix}\right)$. We also recorded the transmitted light at the output of the foam with an imaging diagnostic on an Andor Charged Coupled Device (CCD), on a streak camera and on a photodiode. The use of $\mathrm{Cu}$-doped or $\mathrm{Cl}$-doped foams allowed us to access the electron temperature through a Von Hamos spectrometer with a Bragg crystal. Finally we used a pinhole coupled with an Andor X CCD for plasma imaging. 

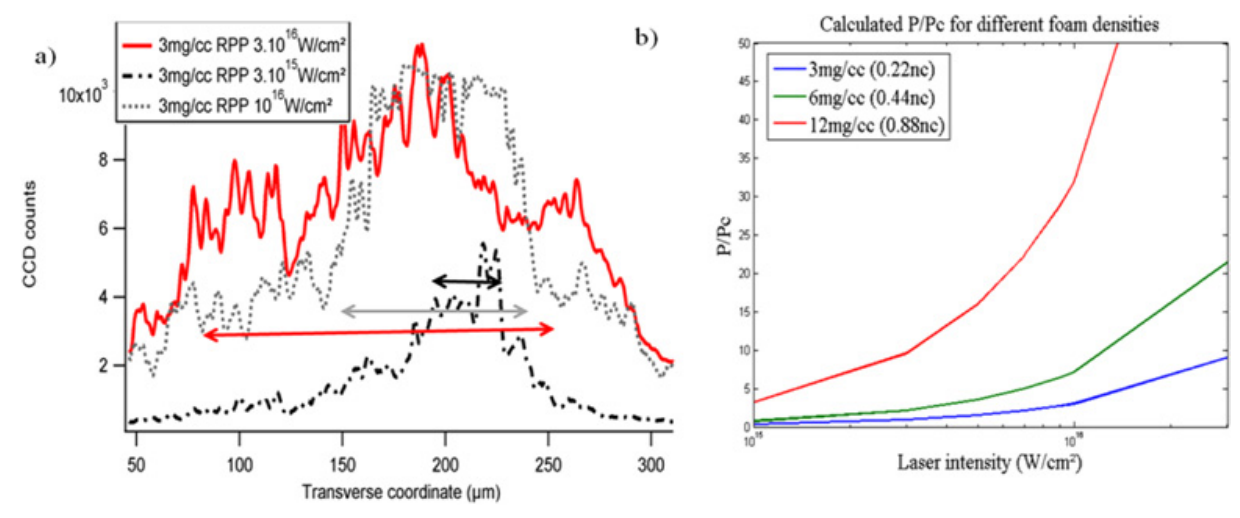

Figure 2. a) Picosecond beam spatial intensity distribution along the horizontal direction at the output of the foam and $\mathrm{b}) \mathrm{P} / \mathrm{P}_{\mathrm{c}}$ for a foam density of 3-6-12 mg versus the laser intensity.

\subsection{Experimental results}

The plasma conditions after one nanosecond of the preforming pulse are known from hydrodynamic simulations. They compare well with the temperature found with the Von Hamos spectrometer. To ensure that the picosecond beam was irradiating a fully ionized foam, we adjusted the delay between the two pulses and the depth along the $y$ axis in the foam. $\mathrm{CH}$ foams of different densities allow us to match part of the exponential density profile expected after the compression stage of a shock ignition target. Then, we used multiple foams target to recreate the whole coronal plasma electron density profile that the spike has to go through.

We studied the effect of the laser intensity varying in the range $3.10^{15} \mathrm{~W} / \mathrm{cm}^{2}$ to $3.10^{16} \mathrm{~W} / \mathrm{cm}^{2}$ on SBS, SRS and filamentation. Figure 2a shows horizontal cuts of the 2D intensity profile obtained with the Andor CCD at the output of the foam, for three intensities. The maximum of the incoming intensity profile (measured without the foam) is around $240 \mu \mathrm{m}$ and the signal FWHM is $50 \mu \mathrm{m}$. We clearly observe spreading of the beam when the intensity increases. For the weakest intensity the focal spot is the same as the incident one, but for $\mathrm{I}=10^{16} \mathrm{~W} / \mathrm{cm}^{2}$, the beam starts spreading and finally for the greatest intensity, the self focusing effect is stronger and the focal spot is more than three times wider than the incident one. As expected we also observed that the energy transmitted decreases when the intensity decreases and a slight deviation of the beam was found. Figure $2 b$ shows the dependence of the $\mathrm{P} / \mathrm{P}_{\mathrm{c}}$ values for the same intensity range as in Fig. 2a considering three different densities. The $3 \mathrm{mg} / \mathrm{cc}$ density has an important $\mathrm{P} / \mathrm{P}_{\mathrm{c}}$ only for the high intensity part of the SI range, the $6 \mathrm{mg} / \mathrm{cc}$ foam $\mathrm{P} / \mathrm{P}_{\mathrm{c}}$ is growing faster and for $12 \mathrm{mg} / \mathrm{cc} \mathrm{P} / \mathrm{P}_{\mathrm{c}}$ is well above 1 for the whole intensity range. As expected, the effect of filamentation is stronger when the foam density is increased.

For the same shots, we found that the SRS spectra are getting wider as the intensity is increasing as shown in Fig. 3. For the lower intensity case, the SRS is emitted from $800 \mathrm{~nm}$ to $900 \mathrm{~nm}$, then for greater intensity cases, it spreads from $570 \mathrm{~nm}$ to $900 \mathrm{~nm}$. Neglecting the temperature, the electron density where the SRS is localized is related to the emission wavelength by the relation:

$$
n_{e} / n_{c} \alpha\left(1-\lambda_{\text {laser }} / \lambda_{\mathrm{SRS}}\right)^{2} .
$$

The broadening observed in the backscattered spectrum indicates that SRS develops also at lower densities when the intensity increases. Due to filamentation, the laser creates holes in the density profile and therefore modifies the emitted spectrum which has lower wavelengths than when filamentation is negligible. 


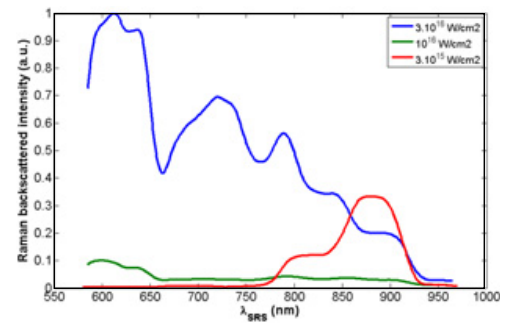

Figure 3. SRS spectra broadening for increasing laser intensity.

\section{CONCLUSION}

In the shock ignition scheme, the shock pulse needs to propagate through a long and hot plasma. This long plasma is expected to be well represented by an exponential density profile of $300-750 \mu \mathrm{m}$ scalelength and the electron temperature $2-4 \mathrm{keV}$.

We have presented in this paper some results for the experiment conducted on the LULI2000 using preformed $\mathrm{CH}$ foams and a $5 \mathrm{ps} 2 \omega 15 \mathrm{~J}$, interacting beam. Concerning the parametric instabilities, these conditions are relevant for the SI scheme spike coupling with the coronal plasma. We observed significant spreading of the beam through the foam due to filamentation for the high intensity case of SI. The analysis of the results is underway and we are looking into other parameters than the intensity like foam density, length etc... Multiple foam targets are also to be analyzed. At the same time, we are conducting hydrodynamic simulations to obtain the hydrodynamic conditions that we reached and interaction simulations to calculate the expected reflectivities. To compare our experiment to shock ignition scheme, one as to keep in mind that no SSD (smoothing by spectral dispersion) technique is used in our experiment. SSD is expected to reduce SBS significantly [11]. We are also looking at interaction in colder plasmas than expected in SI scheme $(\sim 1 \mathrm{keV})$ that should affect beam absorption. Finally, the pulse duration is only 5 ps to be able to change the intensity on one order of magnitude which is shorter than the spike length $(\sim 400 \mathrm{ps})$.

\section{References}

[1] R. Betti et al. Phys. Rev. Lett. 98, 155001 (2007)

[2] X. Ribeyre et al. Plasma Phys. Control. Fusion 51, 015013 (2009)

[3] S. Depierreux et al. Phys. Rev. Lett. 102, 195005 (2009)

[4] S. Depierreux et al. Phys. Rev. Lett. 103, 115001 (2009)

[5] D.T. Michel et al. Phys. Rev. Lett. 103, 115001 (2010)

[6] S. P. Regan et al. Phys. Plasmas 6, 2072 (1999)

[7] O. Klimo et al. Plasma Phys. Control. Fusion 52, 055013 (2010)

[8] C. Riconda et al. Phys. Plasmas 18, 092701 (2011)

[9] O. Klimo et al. Phys. Plasmas 18, 082709 (2011)

[10] S. Depierreux et al, Plasma Phys. Control. Fusion 53, 124034 (2011)

[11] P.E. Masson-Laborde et al., submitted to Phys. Rev. Lett. 